\title{
Children's Trust Beliefs in Others and Trusting Behavior in Peer Interaction
}

\author{
Ken J. Rotenberg, ${ }^{1}$ Serena Petrocchi, ${ }^{2}$ Flavia Lecciso, ${ }^{2}$ and Antonella Marchetti ${ }^{3}$ \\ ${ }^{1}$ School of Psychology, Keele University, Keele, Newcastle-Under-Lyme, Staffordshire ST5 5BG, UK \\ ${ }^{2}$ Universita' del Salento, Cittadella della Ricerca, Strada Statale. n.7, Km 7+300 per Mesagne, 72100 Brindisi, Italy \\ ${ }^{3}$ Universita' Cattolica del Sacro Cuore, Largo Gemelli 1, 20123 Milano, Italy \\ Correspondence should be addressed to Ken J. Rotenberg; k.j.rotenberg@keele.ac.uk
}

Received 5 March 2013; Revised 25 September 2013; Accepted 30 October 2013

Academic Editor: Andrew N. Meltzoff

Copyright (C) 2013 Ken J. Rotenberg et al. This is an open access article distributed under the Creative Commons Attribution License, which permits unrestricted use, distribution, and reproduction in any medium, provided the original work is properly cited.

\begin{abstract}
The relation between children's trust beliefs and trusting behavior in peer interaction was examined. One hundred and 5 Italian children ( 54 boys; mean age $=10$ years -7 months) completed standardized scales of reliability (i.e., promise keeping) trust beliefs in parents and peers. The children participated in mixed-motive interactions with classmates which assessed behavior-dependent reliability trust on peers. The children's reliability trustworthiness towards peers/classmates was assessed by peer reports. The SEM analyses supported the hypothesized model by showing: (1) a path between trust beliefs in parents and trust beliefs in peers; (2) paths between both types of trust beliefs and behavior-dependent trust on peers; (3) a path between behavior-dependent trust in peers and trustworthiness towards peers. Trust beliefs in peers were found to mediate the relation between trust beliefs in parents and behaviordependent trust in peers. The findings yielded support for the basis, domain, and target trust framework and attachment theory.
\end{abstract}

\section{Introduction}

Children's trust beliefs and trusting behavior play a crucial role in their psychosocial functioning [1-4]. There is, however, an important gap in our knowledge. There is a lack of evidence regarding the relation between children's trust beliefs in others (i.e., parents and peers) and the children's trusting behavior in peer interactions. Research has not adequately examined: (a) the precise relation between children's trust beliefs in peers and trusting behavior in peer interaction and (b) the relation between children's trust beliefs in parents and that trusting behavior. The current study was designed to redress that gap in our knowledge. It was guided by the basis, domain, and target (BDT) interpersonal trust framework [3] and attachment theory [5].

\section{The BDT Interpersonal Trust Framework}

The BDT interpersonal trust framework $[3,6]$ comprises 3 Bases (reliability, honesty, and emotional) $\times 3$ Domains (cognitive/affective, behavior-dependent, and behaviorenacting) $\times 2$ Target Dimensions (specificity and familiarity). The framework includes the following three bases of trust: (a) reliability, which refers to a person fulfilling his or her word and promise; (b) emotional trust, which refers to a person refraining from causing emotional harm, such as being receptive to disclosures, maintaining confidentiality of them, refraining from criticism, and avoiding acts that elicit embarrassment; and (c) honesty, which refers to a person telling the truth and engaging in behaviors that are guided by benign rather than malicious intent and by genuine rather than manipulative strategies. The three domains comprise: (a) cognitive/affective which entails individuals' beliefs and feelings that others demonstrate the three bases of trust (e.g., believes that others keep promises); (b) behavior-dependent trust which entails individuals behaviorally relying on others to act in a trusting fashion as per the three bases of trust (e.g., depends on another to keep a promise), and (c) behavior-enacting trust which entails individuals behaviorally engaging in the three bases of trust (e.g., keeps promises). For ease of expression, the term 


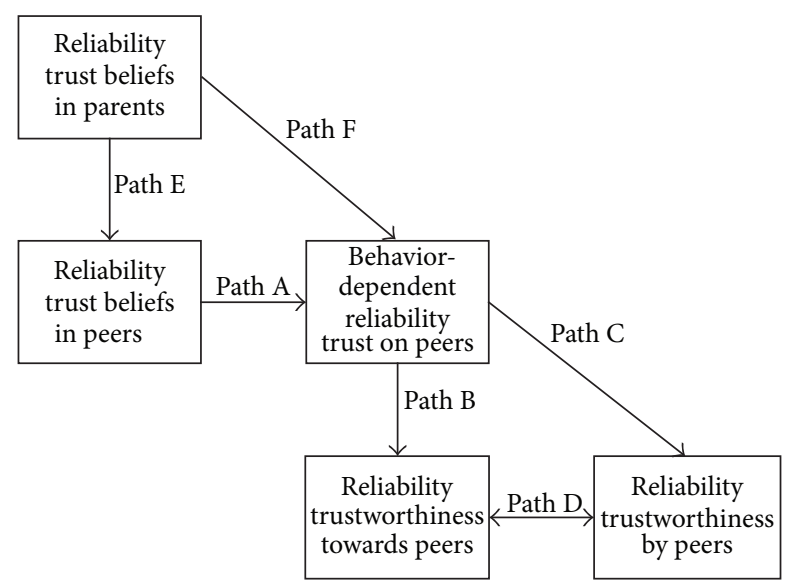

FIgURE 1: Hypothesized model.

trust beliefs will be used to describe the cognitive-affective domain and term trustworthiness will be used to describe the behavior-enacting domain. The BDT framework includes the specificity dimension of the target of trust (ranging from general category to a specific person) and familiarity of the target of trust (ranging from slightly familiar to highly familiar). According to the BDT framework, trust is the result of reciprocal processes whereby individuals demonstrate matching patterns of trust from dyadic interactions and thus establish a common social history with others.

For over three decades trust beliefs and trustworthiness have been assessed from perceptions or reports of promise keeping - the reliability basis of trust-both in children [7, 8] and in adults (see $[6,9,10]$ ). Also, peer reports have been used to assess children's reliability trustworthiness (towards classmates) because peers are uniquely able to observe natural occurring patterns of keeping promises (see [11]). For example, a child's fulfillment of a promise to a classmate often extends across time/social context (e.g., the child promises to meet a classmate after school) and, as a consequence, peers have an advantage over others (e.g., teachers and adult experimenters) in observing such behavior. Therefore the current research focused on reliability trust beliefs/trustworthiness and utilized peer reports of trustworthiness. The BDT framework, when combined with attachment theory, leads to the expectation that there are two types of linkages between children's trust beliefs in others and trusting behavior in interactions with peers: (1) proximal and (2) distal.

2.1. Proximal Link: Peer Interactions. According to the BDT trust framework, a sequence of interactions between children and peers results in a proximal link between trust beliefs in peers and trusting behavior with peers. Figure 1 depicts Paths A, B, C, and D which correspond to that hypothesis. According to the BDT trust framework, there should be a direct link between how much children believe that peers keep promises (reliability trust beliefs in peers) and the extent to which children depend on peers to fulfill promises (behavior-dependent reliability trust on peers; see Path A). This is an extension of the principle that there is a close relation between trust beliefs and trusting behavior regarding the same basis of trust (i.e., reliability) and same target of trust (i.e., peers). As partial support for that hypothesis, Rotenberg et al. [12] found an association between children's reliability trust beliefs on peers and their behavior-dependent reliability trust on peers as demonstrated by cooperating as promised in a mixed-motive game.

According to the BDT trust framework, there should be a link between children's behavior-dependent reliability trust and peers' reliability trustworthiness towards the children (Path C). This path is exemplified by the following example. Consider a situation in which a child and a classmate plan to engage in a joint activity of going to movies after school at a specified time. The child goes to the movies at the agreedupon time and therefore demonstrates behavior-dependent reliability trust in a peer. Because of the norm of responsibility, the peer also shows up at the agreed-upon time thus fulfilling his/her promise and demonstrating reliability trustworthiness towards the child. As a result of this process, there is a link between children's behavior-dependent reliability trust on peers and reliability trustworthiness towards the children. This hypothesized relation is shown as Path $\mathrm{C}$ in Figure 1.

Finally, there is evidence that reciprocity guides peer interactions (see [13]) including exchanges of trustworthiness between children and their peers as hypothesized by the BDT trust framework $[7,14]$. Guided by this principle, it was expected that there would be paths between: (1) children's reliability trustworthiness towards peers and peers' reliability trustworthiness towards the children due to reciprocity per se (see Path D) and (2) children's behavior-dependent reliability trust on peers and children's reliability trustworthiness as a consequence of the former relation (see Path B). There is a scarcity of research regarding the latter relation and its examination in the current study provided a unique test of the BDT trust framework.

Paths C and D were not examined in the current study because they overlapped with the measure of children' behavior-dependent reliability trust on peers. Children's behavior-dependent reliability trust on peers is conventionally assessed from the children's willingness to reciprocate the promise and intended behavior of peers (see [11]). As a consequence, behavior-dependent reliability trust on peers includes a form of reciprocity of trustworthiness between children and their peers and therefore overlaps with the corresponding paths.

2.2. Distal Link: Children's Trust Beliefs in Parents. The distal link comprises the relation between children's trust beliefs in parents and both children's trust beliefs in peers and trusting behavior in peer interaction. This linkage is derived from attachment theory (IWM; $[5,15,16])$ which posits that children establish an internal working model which affects their later psychosocial functioning. Attachment theorists have proposed that securely as opposed to insecurely attached children develop an IWM comprising social expectations characterized by a sense of trust in others and by positive thoughts regarding the intentions of other people's behaviors $[17,18]$. The IWM provides children with cognitive structure for their trust beliefs in others such as peers which, according to attachment theory, affects the quality of their peer interaction. 
Guided by the attachment theory, Paths E and F (shown in Figure 1) were expected regarding the reliability basis of trust. The distal paths subsume the proximal links paths that were previously described. It was hypothesized that there would be a relation (see Path E) between children's reliability trust beliefs in parents and the children's reliability trust beliefs in peers as a result of the IWM. Also, it was hypothesized that there would be a relation (see Path F) between children's reliability trust beliefs in parents and their behavior-dependent reliability trust on peers as a result of the social consequences of children's IWM. Furthermore, it was expected that the latter relation would be mediated by children's reliability trust beliefs in peers. This latter path and mediation reflected the principle that children's reliability trust beliefs in parents (as part of their IWM) would affect children's behavior-dependent reliability trust on peers because, in part, of its effects on children's reliability trust beliefs in peers.

Research has yielded some support for the hypothesized paths derived from attachment theory. Researchers have identified children's representation of trust in their caregivers as part of their IWM [19-21]. Furthermore, researchers have found that children's trust in caregivers (primarily mothers) is associated with their visual attention to caregivers [22] and reliance on them as a source of information [23]. Also, researchers have found that children's trust beliefs in parents are associated with their trust beliefs in peers [21, 24, 25]. Finally, meta-analyses (e.g., [26]) show that there is a relation between the quality of children's attachment with parents and peer competence, which range in magnitude from a small to a modest effect size. Nevertheless, there is a lack of research that specifically addresses the paths depicted in Figure 1 between children's trust beliefs in parents and trusting behaviors in peer interaction. The current study was designed to examine those paths in a sample of Italian children.

\section{What Is Trusting Behavior?}

A substantial body of research is guided by the principle that trusting behavior is essentially cooperative in nature: individuals trust others by cooperating in response to promised cooperation ([27]; see [4]). A different conceptualization of trusting behavior is advanced by BDT trust framework. According to the BDT trust framework, reliability trusting behavior comprises individuals: (a) depending on others to fulfill promises to cooperate or compete and (b) the reciprocation of those types of promises and behaviors. In naturally occurring peer interactions children are inclined to cooperate with peers who promise to cooperate and compete with peers who promise to compete (see $[28,29])$. We expected that children's trust beliefs in parents/peers would be associated with behaviorally depending on peers by promised cooperation when peers had promised to cooperate and by promised competition when peers had promised to compete.

\section{Children's Trust Beliefs and Their IWM}

A priority in the current study was the measurement of children's trust in parents as a component of the IWM. The
People on My Life scale (PIML; [30]) is the only measure (to our knowledge) designed to assess the IWM during middle childhood that includes a separate subscale to measure trust in parents. This subscale includes items such as "My parent respects my feelings" and "I trust my parents". Although the PIML scale and the trust subscale are valuable, there are several limitations with the latter for the current study. Specifically, the notions of "trust" and "respect" are abstract and it is unclear what children mean by those judgments. Furthermore, the current research was designed to examine the specific relation between children's beliefs in the reliability (promise keeping) of their parents and the children's reliability trusting behavior in peer interactions. In order to resolve this issue, we employed the children's generalized trust belief scale (CGTB; [25]) as a measure of that component of the trust in the IWM because it included reliability trust beliefs in mothers and fathers subscales. This subscale assessed children's concrete expectations regarding the extent to which parents fulfill their promises to their offspring.

According to Greenberg and his colleagues [19, 30], trust beliefs in parents in the IWM entail children's belief that parents show respect for their feelings and sensitivity to those feelings as part of cognitive-affective regulatory parent-child interaction (i.e., attachment). Similar processes are evident when parents make and fulfill promises to children (i.e., the reliability basis of trust). By fulfilling promises to children, parents are teaching the children to delay gratification which involves linking their internal states (i.e., feelings and desires) to outcomes across time as a result of parental communication and behavior. When promises are fulfilled, parents demonstrate to their children respect for, and sensitivity to, their feelings that promote children's cognitive-affective regulation as part of parent-child relationships. For these reasons, we used the reliability trust in mothers and fathers subscales of the CGTB to assess the trust component of the IWM. Furthermore, researchers (see [30]) have emphasized the importance of separately assessing trust in mothers and trust in fathers as part of the IWM because of the potential differential attachment to the two parents. Also, Ridenour et al. [30] have proposed that the trust component of IWM is a central cognition during middle childhood (10 to 12 years) and affects their attachment to peers and peer relationships. Therefore we examined the hypothesized associations: (a) separately for trust beliefs in mothers and trust beliefs in fathers and (b) during the period of middle childhood (e.g., 10 years).

4.1. Gender Differences. Research has shown that girls demonstrate greater trustworthiness (as reported by peers) than do boys in the UK [11, 31]. One account of the gender differences is that: (a) inhibitory control is greater in girls than in boys [32] and (b) inhibitory control contributes to trustworthiness [11, 31]. We examined whether the observed gender differences in reliability trustworthiness towards peers is replicable, notably for children from Italy.

4.2. Gender as a Moderator of the Hypothesized Paths and Observed Relations. Researchers have found that gender moderates the relation between psychological problems (e.g., 
loneliness) and trust beliefs/behavior: stronger relations were found in girls than in boys [11]. We examined whether or not gender moderated the hypothesized paths and relations between children's trust beliefs in others and trusting behaviors towards peers.

\section{Method}

5.1. Participants. The sample was 105 children (54 boys) who were enrolled in 4 th and 5 th grade classrooms in Italy. The mean age of the participants was 10 years- 7 months and the age ranged from 10 years- 1 month to 11 years -3 months. The participants were drawn from 4 schools that primarily served middle class neighborhoods. The size of the classes ranged from 12 to 20 pupils.

\subsection{Measures}

5.2.1. Reliability Trust Beliefs in Parents. This was assessed by the mother and father reliability basis subscales of the children's generalized trust belief scale (CGTB; [25]) that had been translated into Italian (i.e., the ICGTB). The English CGTB has been found to show construct validity by factor analyses and correlations with other comparable measures of trust beliefs [25]. The Italian mother and father reliability trust beliefs basis subscales were composed of four items (two per each target). Each item describes a short vignette depicting a parent (mother or father) who made a promise to a child (i.e., the protagonist) who was the same gender as the participant. The participant was required to imagine being the protagonist in the story and then required to judge, on a 5-point Likert scale (ranging from 1-very unlikely to 5very likely), how likely the parent would keep promises he/she had made. The four items were combined to obtain a measure of children's reliability trust beliefs in parents which showed acceptable internal consistency, $\alpha=0.57$, given the limited number of items in the scale. Preliminary confirmatory factor analyses showed that these measures assessed the reliability basis of trust beliefs as distinct from the emotional and honesty bases of trust beliefs.

5.2.2. Reliability Trust Beliefs in Peers. This was assessed by the measure developed by Rotenberg and his colleagues $[25,33]$. Participants rated on a 5-point scale, ranging from 1-never to 5-always, how often each classmate in his or her class keeps promises he/she had made. The participant's promise keeping ratings were summed across classmates (and averaged) to yield a measure of his or her reliability trust beliefs in peers. Higher scores on the measure corresponded to greater reliability trust beliefs in peers. Research yields evidence for the reliability and validity of this measure of trust belief in peers. Betts and Rotenberg [7] found that this measure demonstrated acceptable levels of internal consistency as evidenced by statistically significant "actor effects" on that measure. As support for validity, Rotenberg et al. [11] found that this measure was positively correlated with the peer subscale of the children's generalized trust belief scale across a year span.
5.2.3. Reliability Trustworthiness towards Peers. This was assessed by the measure established by Rotenberg and his colleagues $[7,11]$ that draws upon the (preceding) children's ratings of the extent to which classmates keep promises. Reliability trustworthiness towards peers was calculated by the ratings of promise keeping that each participant received from all his or her classmates and divided by the number of classmates judging to adjust for class size. Higher scores on this measure corresponded to greater reliability trustworthiness towards peers, as reported by peers.

Research has yielded evidence for the reliability and validity of this measure of trustworthiness towards peers. Acceptable levels of stability of this trustworthiness measure have been found across a one-year span in young children, $r(209)=.69, P<.001$ [31] and in older elementary school children, $r(288)=.49, P<.001[11$, Study 2]. As evidence for the internal consistency of this measure, Betts and Rotenberg [7] found significant "Partner Effects" in round robin analyses which show that peers demonstrate considerable agreement in their reports of promise keeping of individual children. In support of construct validity, researchers have found a statistically significant correspondence between peers' reports (this measure) and teachers' reports of the extent to which children keep promises to peers [34].

5.2.4. Behavior-Dependent Reliability Trust on Peers. Each participant engaged in a mixed-motive interaction (MMI) game-a version of the Prisoner's Dilemma game-with same-sex classmates. Each participant was presented a light apparatus composed of a red light and a green light. Those lights were connected to corresponding buttons that the participant pressed in order to provide his/her selection. The contingencies (i.e., payoffs) of the MMI game were the following: (1) if both players selected the green light, then they both received 30 points; (2) if one player selected red and the other selected green, then the former player received 40 points and the latter player received 10 points; and (3) if both players selected red then they each received 5 points. The participant was informed that the number of points he/she won could be exchanged for an equivalent number of sweets (candies). As a result of these payoffs, the selection of the green light was a cooperative choice and the selection of the red light was a competitive choice. In response to questioning, all the participants demonstrated an understanding of the game and the contingencies of play (i.e., they identified the different rewards obtained from the different choices).

Two sets of trials of the MMI game were completed for each participant. In one set of trials, the tester identified a classmate by name from the list. The participant was then told that the classmate had said that he/she would select a green light (i.e., promised to cooperate). The tester then asked the participant: (1) to verbally state what light he/she would say to the classmate as a choice (i.e., a promise) and (2) then to identify what light he/she (the participant) would choose by pushing down the corresponding button on the apparatus. This procedure was repeated for each classmate on the list.

The same procedure was followed in another set of trials, except that the participant was told that the classmate had said that he or she would select a red light (i.e., promised 
to compete). Again, the entire procedure was repeated for each classmate on the list. The order of the two sets of trials (i.e., promised to cooperate versus promised to compete) was counterbalanced across the participants. In addition, the order of the names of the classmates was randomized across the different participants in order to eliminate any order of classmate effect.

The participants' promise and choice in response to each type of classmates' promises (cooperate versus to compete) were coded in the following fashion.

(1) The combination in which the classmate promised to cooperate and participant promised to cooperate and then chose to cooperate was coded as high in behavior-dependent reliability trust on peers. This was coded in that fashion because the participant demonstrated a willingness to depend on the classmate's promise by similarly promising to cooperate and then engaging in that behavior.

(2) The combination in which the classmate promised to cooperate and the participant promised to cooperate but chose to compete was coded as low in behavior-dependent reliability trust on peers. This was coded in that fashion because the participant demonstrated an unwillingness to depend on the classmate's promise. The participant initially matched the classmate's promise but engaged in behavior contrary to it.

(3) The combination in which the classmate promised to compete and the participant promised to compete but chose to cooperate was coded as low in behaviordependent reliability trust on peers. This was coded in that fashion because the participant demonstrated an unwillingness to depend on the classmate's promise. In this case, the participant initially matched the classmate's promise to compete but then engaged in behavior contrary to it.

(4) The combination in which the classmate promised to compete and the participant promised to compete and then competed was coded as high in behaviordependent reliability trust on peers. This was coded in that fashion because the participant was willing to depend on the classmate's promise by similarly promising to compete and then engaged in that behavior.

A preliminary set of correlations were carried out to guide the construction of the measure of behavior-dependent reliability trust on peers. These analyses confirmed that: (1) the two high behavior-dependent reliability trust combinations were correlated, $r(93)=0.64, P<0.001$, and (2) the two low behavior-dependent reliability trust combinations were correlated, $r(93)=0.57, P<0.001$. Also, as expected there were negative correlations between each of the two high behavior-dependent reliability trust combinations and each of the two low behavior-dependent reliability trust combinations, $r \mathrm{~s}(93)=-0.53$ to $-0.64, P \mathrm{~s}<0.001$. The behavior-dependent reliability trust on peers measure was calculated by summing the two high behavior-dependent trust combinations and subtracting from it the sum of the two low behavior-dependent reliability trust combinations. The resulting scores were subjected to a log transformation in order to normalize the distribution. Higher scores on the resulting measure denoted greater behavior-dependent reliability trust on peers. It should be pointed out that additional correlations confirmed that the other combinations of promised cooperation and competition in the MMI game did not appreciably contribute to, or detract from, any of the relations reported in this paper.

5.3. Procedure. The participants were tested by a female adult in two separate sessions. In the first session, the participants were individually: (a) administered the reliability trust beliefs in peers measure, (b) administered the reliability trustworthiness towards peers measure, and (c) engaged in MMI. This session was conducted individually (away from classmates) because it pertained to judgments of, and behaviors towards, classmates. In the second testing session, participants were administered in small same-sex groups (5 or 6) the reliability trust beliefs in parents measure. Great care was taken in the second testing session to ensure that the children completed the measure independently and privately. Standardized instructions were given to encourage participants to reply honestly and highlighting that this was not a test and there were no right or wrong answers.

\section{Results}

6.1. Correlations between the Measures. A preliminary set of correlations was carried out among the variables separately for reliability trust beliefs in mothers and reliability trust beliefs in fathers. The correlations with trust beliefs in mothers and trust beliefs in fathers were not statistically different (the $z$ s were at $P>0.05$ ) and the correlations were all in the same direction (i.e., all were positive). As a consequence the reliability trust beliefs in mothers and those in fathers were summed (and averaged) to serve as a reliability trust belief in parents measure for the primary analyses. The correlations between the measures (with means and SDs) are shown in Table 1. The table shows that consistent with the hypothesized paths: (a) reliability trust beliefs in parents were correlated with reliability trust beliefs in peers, (b) both of those trust belief measures were correlated with behavior-dependent reliability trust on peers, and (c) behavior-dependent reliability trust on peers was correlated with reliability trustworthiness towards peers. In addition, reliability trust beliefs in parents were correlated with reliability trustworthiness towards peers.

6.2. Structural Equation Modeling (SEM) Analyses. The hypothesized paths and model were tested by SEM analyses. The results confirmed that the hypothesized model (shown in Figure 2) was a good fit of the data with $\chi^{2}(2)=3.61$, $P=0.16$, comparative fit index $(\mathrm{CFI})=0.91$, and root mean square error of approximation (RMSEA) $=0.088$. Researchers regard that a model is a good fit when there is a nonsignificant $\chi^{2}$, the CFI $>0.90$, and RMSEA is 0.05 to 0.08 or less (see 
TABLE 1: Correlations between the measures (with means and SDs).

\begin{tabular}{|c|c|c|c|c|c|}
\hline Measure & Mean & SD & RTBP & BDRTP & RTWTP \\
\hline Reliability trust beliefs in parents & 8.70 & 1.30 & $0.29^{* *}$ & $0.27^{* *}$ & $0.21^{*}$ \\
\hline Reliability trust beliefs in peers (RTBP) & 3.49 & 0.69 & & $0.26^{*}$ & 0.17 \\
\hline Behavior-dependent reliability trust on peers (BDRTP) & 0.25 & 0.18 & & & $0.20^{*}$ \\
\hline Reliability trustworthiness towards peers (RTWTP) & 3.39 & 0.59 & & & \\
\hline
\end{tabular}

${ }^{*} P<.05$ and ${ }^{* *} P<.01$, the dfs ranged from 96 to 103.

[35]). The RMSEA was marginally higher than desired but the indices converge in demonstrating that the model is a good fit. The SEM analyses yielded evidence for the four hypothesized paths. There were paths between: (a) reliability trust beliefs in parents and reliability trust beliefs in peers (Path E); (b) reliability trust beliefs in parents and behavior-dependent reliability trust on peers (Path F) and (b) reliability trust beliefs in peers and behavior-dependent reliability trust on peers (Path A) and (c) behavior-dependent reliability trust on peers and reliability trustworthiness towards peers (Path B).

6.3. A Test of Hypothesized Mediation. According to the model, reliability trust beliefs in peers (RTB-PEERS) should serve as a mediator between trust beliefs in parents (RTBPARENTS) and behavior-dependent reliability trust on peers (BDRT-PEERS). The expected mediation was assessed by the 5,000 bootstrap resample procedure recommended by Preacher and Hayes [36]. When RTB-PARENTS was the predictor, the test of simple mediation yielded significant: (1) effects of RTB-PARENTS on BDRT-PEERS, $b=1.88$ $(\mathrm{SE}=0.68), t(96)=2.76, P=0.007,(2)$ effects of RTBPEERS on BDRT-PEERS, $b=0.97(\mathrm{SE}=0.37), t(96)=2.67$, $P=0.011$, and (c) indirect effects of RTB-PARENTS on BDRT-PEERS through RTB-PEERS, $b=0.45$ ( $\mathrm{SE}=0.18$ ), $t(96)=2.47, P=0.015$. The bootstrap results for the $95 \%$ confidence level were 0.024 for the lower confidence level and 0.94 for the upper confidence level. Because zero did not fall with this range, the indirect effect of BDRT-PEERS attained significance at $P<0.05$. The findings yielded support for the hypothesis that reliability trust beliefs in peers served as a mediator between children's reliability trust beliefs in parents and behavior-dependent reliability on peers.

6.4. Gender Differences. Individual $t$-tests on the measures showed, as hypothesized, that there were gender differences in reliability trustworthiness towards peers, $t(1,103)=4.38$, $P=0.039$. As expected, higher reliability trustworthiness towards peers was demonstrated by girls, $M=3.50$ than did by boys, $M=3.27$.

6.5. Gender as a Moderator of the Observed Correlations and Paths. Hierarchical regression analyses (HRAs) were carried out to examine whether gender moderated the observed correlations shown in Table 1. Two measures were selected with one serving as the predictor (which was centered) and the other serving as the dependent measure. Gender served as potential moderating variable and it was dummy coded. In a given HRA, the predictors were (Step 1) the predictor

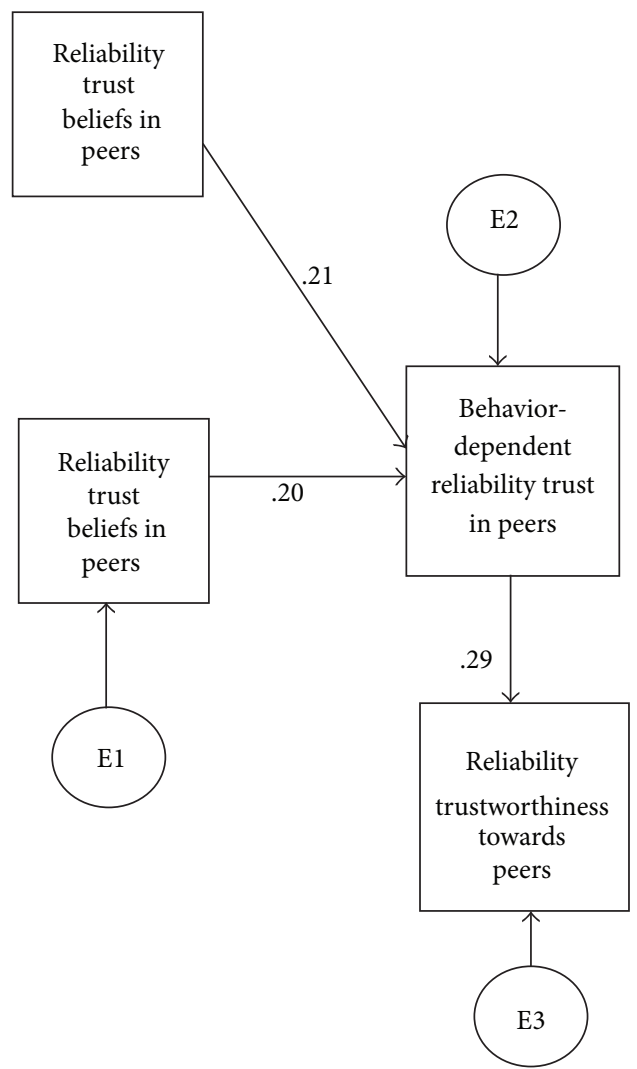

Figure 2: SEM Analysis of the Hypothesized Model. All the paths attained statistical significance.

measure, (Step 2) gender, and (Step 3) the predictor $*$ gender term (see [37]). The HRAs did not yield a significant measure * gender interaction for any of the dependent measures. As a consequence, gender was not found to moderate the observed relations.

As a test of whether gender served as a moderator of the hypothesized model, the SEM (as shown in Figure 2) was carried out with the paths constrained by gender (see [38]). The SEM constrained by gender yielded $\chi^{2}(4)=0.97$, $P=0.95$ which showed that gender did not moderate the observed paths.

\section{Discussion}

The findings yielded support for the hypothesized model. The SEM analyses confirmed that the model was a good fit, confirming: (1) a path between children's reliability trust 
beliefs in parents and children's reliability trust beliefs in peers (Path E); (2) paths between reliability trust beliefs in both parents and peers and behavior-dependent reliability trust on peers (Paths F and A, resp.); (3) a path between children's behavior-dependent reliability trust on peers and reliability trustworthiness (Path B). A corresponding pattern of correlations was observed. As expected, children's reliability trust beliefs in peers were found to serve as a mediator between children's reliability trust beliefs in parents and behavior-dependent reliability on peers. Finally, as hypothesized, girls demonstrated greater reliability trustworthiness towards peers than did boys.

The current findings yielded support for the proximal links derived from the BDT interpersonal trust framework [3]. The expected paths were found between children's reliability trust beliefs in peers and children's behavior-dependent reliability trust on peers, as well as between the latter and the children's reliability trustworthiness towards peers. The findings complement the association observed by Rotenberg et al. [11] between children's reliability trust beliefs in peers and their behavior-dependent reliability trust on peers by cooperating as promised. The current findings extend the research by showing that children's reliability trust beliefs in peers are associated with reciprocating the promises and intended behaviors of peers who either compete or cooperate as suggested by the BDT interpersonal trust framework [3]. The current findings may be interpreted as showing that children's trust beliefs in peers promote normative patterns of promised cooperation and competition in peer interaction (see $[28,29])$. The approach that diverges from the principle that trusting behavior is essentially cooperative in nature comprising individuals trusting others by co-operating in response to promised cooperation ([27]; see [4]).

The current study uniquely showed a positive relation between children's behavior-dependent reliability trust on peers and reliability trustworthiness towards peers. It is worthwhile to note that those two variables were assessed by very different types of measures: behavior-dependent reliability trust on peers was assessed by children's behavior towards peers in a mixed-motive interaction game whereas reliability trustworthiness was assessed by peer reports. The findings support the hypothesis that the more children depend on peers to fulfill their promises (i.e., behaviordependent reliability trust), the more they are likely to engage in reliability trustworthiness towards peers.

The findings yielded evidence for the hypothesized distal linkages and thus paths between children's reliability trust beliefs in parents and behavior-dependent reliability trust on peers. Also, the findings yielded support for the hypothesis that children's reliability trust beliefs in parents served as a mediator between those two variables. These findings are consistent with the attachment theory guided principles [17, 18] that children's trust beliefs in parents, as part of their IWM, provide the cognitive structure for their trust beliefs in peers and thus affect their trusting behavior with peers as a form of peer competence.

The current findings confirmed the expectation that girls demonstrate greater reliability trustworthiness towards peers than do boys. Studies have shown gender differences in the trustworthiness of children from UK and Canada (see $[11,31]$ ) and those differences were replicated in a sample of children from Italy.

The findings did not show that the observed correlations and paths were moderated by gender. The findings contrast with Rotenberg et al. [11] who found that gender moderates the relation between psychological problems (i.e., loneliness) and trust beliefs/behavior with stronger relations in girls than in boys. The difference in the patterns may be attributed to the fact that Rotenberg et al. [11] examined the relation between psychological problems and trust beliefs/behavior rather than between trust beliefs and behavior in the current study.

\section{Directions for Future Research}

In future, researchers might wish to examine the following three issues. First, the current study examined concurrent relations among trust beliefs and trusting behavior with peers. Longitudinal research is needed to provide clearer evidence for the hypothesized causal relations.

Second, research shows that children's trustworthiness towards peers contributes to the development of peer friendships [11] and, therefore, it would be desirable to promote such behavior in children. In a related fashion, researchers have found that the social functioning in withdrawn children can be promoted by engaging them in adult-lead interventions comprising interactive learning and practice in natural settings with peers (see [39]). Similar interventions might be useful for increasing trustworthiness in children who demonstrate low reliability trustworthiness towards peers. Based on the observed relations, it would be reasonable for adults to prompt and instruct low trustworthy children to behaviorally depend on peers to fulfill promises in natural settings as a means of increasing the children's trustworthiness.

Third, the trust component of the IWM was assessed by reliability trust beliefs in mothers and fathers from the generalized trust belief scale [25]. In future, researchers may wish to examine the relation between those scales and other measures of IWM which include subscales assessing trust in parents-such as the PIML [30]. The current study focused on the relation between children's reliability based trust beliefs and reliability trusting behavior in peer interactions and consequently the currently used measures of trust beliefs were appropriate. Furthermore, it would be worthwhile to examine the relation between the current used measures of trust beliefs in parents and the other components of the IWM such as communication and alienation (see $[19,30])$.

\section{References}

[1] E. H. Erikson, Childhood and Society, Norton, New York, NY, USA, 1963.

[2] P. L. Harris, “Trust," Developmental Science, vol. 10, no. 1, pp. 135-138, 2007.

[3] K. J. Rotenberg, "The conceptualization of interpersonal trust: a basis, domain, and target framework," in Interpersonal Trust During Childhood and Adolescence, K. J. Rotenberg, Ed., pp. 227, Cambridge University Press, New York, NY, USA, 2010. 
[4] J. A. Simpson, "Foundations of interpersonal trust," in Social Psychology: Handbook of Basic Principles, A. W. Kruglanski and E. T. Higgins, Eds., pp. 587-607, Guilford Press, New York, NY, USA, 2nd edition, 2007.

[5] J. Bowlby, Attachment. Attachment and Loss, vol. 1, Basic Books, New York, NY, USA, 1969.

[6] K. J. Rotenberg, "Loneliness and interpersonal trust," Journal of Social and Clinical Psychology, vol. 13, pp. 152-173, 1994.

[7] L. R. Betts and K. J. Rotenberg, "A social relations analysis of children's trust in their peers across the early years of school," Social Development, vol. 17, no. 4, pp. 1039-1055, 2008.

[8] D. J. Hochreich, "A children's scale to measure interpersonal trust," Developmental Psychology, vol. 9, no. 1, article 141, 1973.

[9] J. B. Rotter, "Interpersonal trust, trustworthiness, and gullibility," American Psychologist, vol. 35, no. 1, pp. 1-7, 1980.

[10] K. J. Rotenberg, N. Addis, L. R. Betts et al., "The relation between trust beliefs and loneliness during early childhood, middle childhood, and adulthood," Personality and Social Psychology Bulletin, vol. 36, no. 8, pp. 1086-1100, 2010.

[11] K. J. Rotenberg, P. McDougall, M. J. Boulton, T. Vaillancourt, C. Fox, and S. Hymel, "Cross-sectional and longitudinal relations among peer-reported trustworthiness, social relationships, and psychological adjustment in children and early adolescents from the United Kingdom and Canada," Journal of Experimental Child Psychology, vol. 88, no. 1, pp. 46-67, 2004.

[12] K. J. Rotenberg, K. J. Macdonald, and E. V. King, "The relationship between loneliness and interpersonal trust during middle childhood," Journal of Genetic Psychology, vol. 165, no. 3, pp. 233-249, 2004.

[13] D. D. DeLawyer and S. L. Foster, "The effects of peer relationship on the functions of interpersonal behaviors of children," Journal of Clinical Child Psychology, vol. 15, pp. 127-133, 1986.

[14] K. J. Rotenberg and M. J. Boulton, "The relations between interpersonal trust consistency and quality of peer relationships during childhood," Social Development, vol. 22, no. 2, pp. 225241, 2013.

[15] J. Bowlby, Separation: Anxiety \& Anger. Attachment and Loss, vol. 2, Hogarth Press, London, UK, 1973.

[16] J. Bowlby, Loss: Sadness \& Depression. Attachment and Loss, vol. 3, Hogarth, London, UK, 1980.

[17] D. A. Cohn, "Child-mother attachment of six-year-olds and social competence at school," Child Development, vol. 61, no. 1, pp. 152-162, 1990.

[18] A. F. Lieberman, "Preschoolers' competence with a peer: relations with attachment and peer experience," Child Development, vol. 48, pp. 1277-1287, 1977.

[19] G. C. Armsden and M. T. Greenberg, "The inventory of parent and peer attachment: individual differences and their relationship to psychological well-being in adolescence," Journal of Youth and Adolescence, vol. 16, no. 5, pp. 427-454, 1987.

[20] K. M. Dwyer, "The meaning and measurement of attachment in middle and late childhood," Human Development, vol. 48, no. 3 , pp. 155-182, 2005.

[21] A. Sakai, "Children's sense of trust in significant others: genetic versus environmental contributions and buffer to life stressors," in Interpersonal Trust During Childhood and Adolescence, K. J. Rotenberg, Ed., pp. 56-84, Cambridge University Press, New York, NY, USA, 2010.

[22] G. Bosmans, C. Braet, E. Koster, and R. de Raedt, "Attachment security and attentional breadth toward the attachment figure in middle childhood," Journal of Clinical Child and Adolescent Psychology, vol. 38, no. 6, pp. 872-882, 2009.
[23] K. H. Corriveau, P. L. Harris, E. Meins et al., "Young children's trust in their mother's claims: longitudinal links with attachment security in infancy," Child Development, vol. 80, no. 3, pp. 750-761, 2009.

[24] S. Imber, "Relationship of trust to academic performance," Journal of Personality and Social Psychology, vol. 28, no. 1, pp. 145-150, 1973.

[25] K. J. Rotenberg, C. Fox, S. Green et al., "Construction and validation of a children's interpersonal trust belief scale," British Journal of Developmental Psychology, vol. 23, no. 2, pp. 271-292, 2005.

[26] B. H. Schneider, L. Atkinson, and C. Tardif, "Child-parent attachment and children's peer relations: a quantitative review," Developmental Psychology, vol. 37, no. 1, pp. 86-100, 2001.

[27] H. H. Kelley, J. G. Holmes, N. L. Kerr, H. T. Reis, C. E. Rusbult, and P. A. M. Van Lange, An Atlas of Interpersonal Situations, Cambridge University Press, New York, NY, USA, 2003.

[28] P. M. Averill and T. G. Power, "Parental attitudes and children's experiences in soccer: correlates of effort and enjoyment," International Journal of Behavioral Development, vol. 18, pp. 263-276, 1995.

[29] H. Feldlaufer, C. Midgley, and J. S. Eccles, "Student, teacher, and observer perceptions of the classroom environment before and after the transition to junior high school," The Journal of Early Adolescence, vol. 8, pp. 133-156, 1988.

[30] T. A. Ridenour, M. T. Greenberg, and E. T. Cook, "Structure and validity of people in my life: a self-report measure of attachment in late childhood," Journal of Youth and Adolescence, vol. 35, no. 6, pp. 1037-1053, 2006.

[31] L. R. Betts and K. J. Rotenberg, "Trustworthiness, friendships and self-control: factors that contribute to young children's school adjustment," Infant and Child Development, vol. 16, pp. 491-508, 2007.

[32] K. L. Moilanen, D. S. Shaw, T. J. Dishion, F. Gardner, and M. Wilson, "Predictors of longitudinal growth in inhibitory control in early childhood," Social Development, vol. 19, no. 2, pp. 326347, 2010.

[33] K. J. Rotenberg, "Sex differences in children's trust in peers," Sex Roles, vol. 11, no. 9-10, pp. 953-957, 1984.

[34] K. J. Rotenberg, N. Michalik, N. Eisenberg, and L. R. Betts, "The relations among young children's peer-reported trustworthiness, inhibitory control, and preschool adjustment," Early Childhood Research Quarterly, vol. 23, no. 2, pp. 288-298, 2008.

[35] L. T. Hu and P. M. Bentler, "Evaluating model fit," in Structural Equation Modeling: Concepts, Issues, and Applications, R. H. Hoyle, Ed., pp. 76-99, Sage, Thousand Oaks, Calif, USA, 1995.

[36] K. J. Preacher and A. F. Hayes, "SPSS and SAS procedures for estimating indirect effects in simple mediation models," Behavior Research Methods, Instruments, and Computers, vol. 36, no. 4, pp. 717-731, 2004.

[37] J. Cohen, P. Cohen, S. G. West, and L. S. Aiken, Applied Multiple Regression/Correlation Analysis for the Behavioral Sciences, Lawrence Erlbaum, Mahwah, NJ, USA, 3rd edition, 2003.

[38] B. M. Byrne, Structural Equation Modelling: Basic Concepts, Applications, and Programming, Lawrence Erlbaum Associates, Mahwah, NJ, USA, 2001.

[39] P. A. Wyman, W. Cross, C. H. Brown, Q. Yu, X. Tu, and S. Eberly, "Intervention to strengthen emotional self-regulation in children with emerging mental health problems: proximal impact on school behavior," Journal of Abnormal Child Psychology, vol. 38, no. 5, pp. 707-720, 2010. 

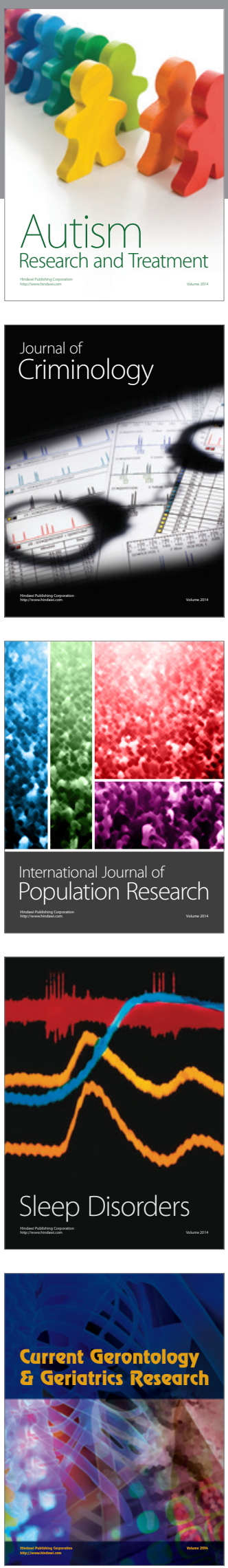
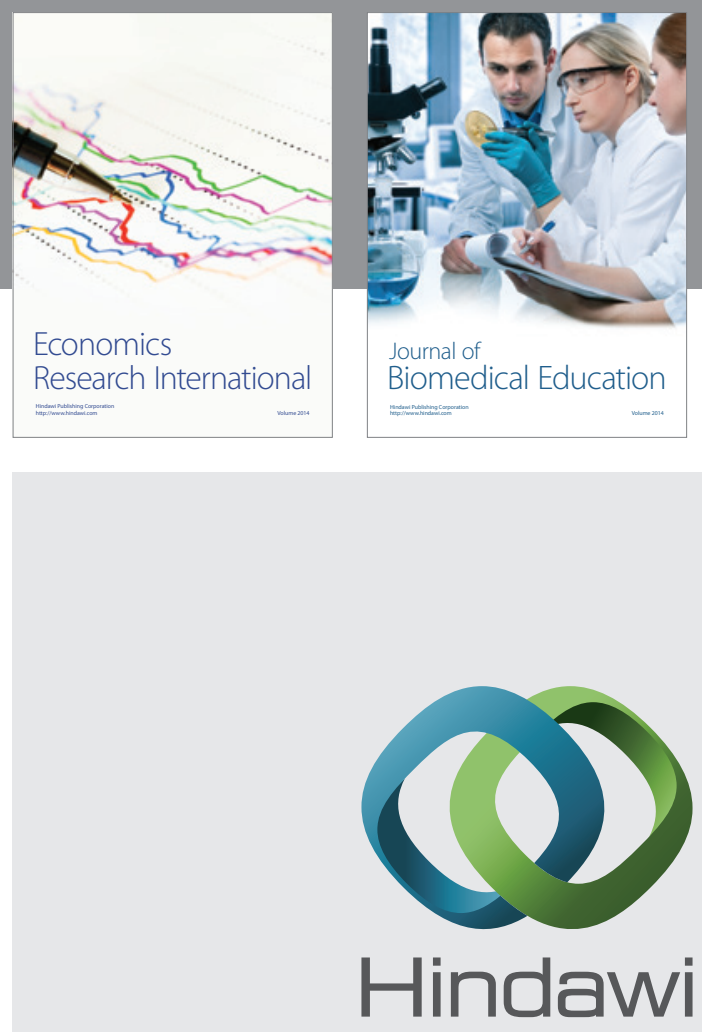

Submit your manuscripts at

http://www.hindawi.com
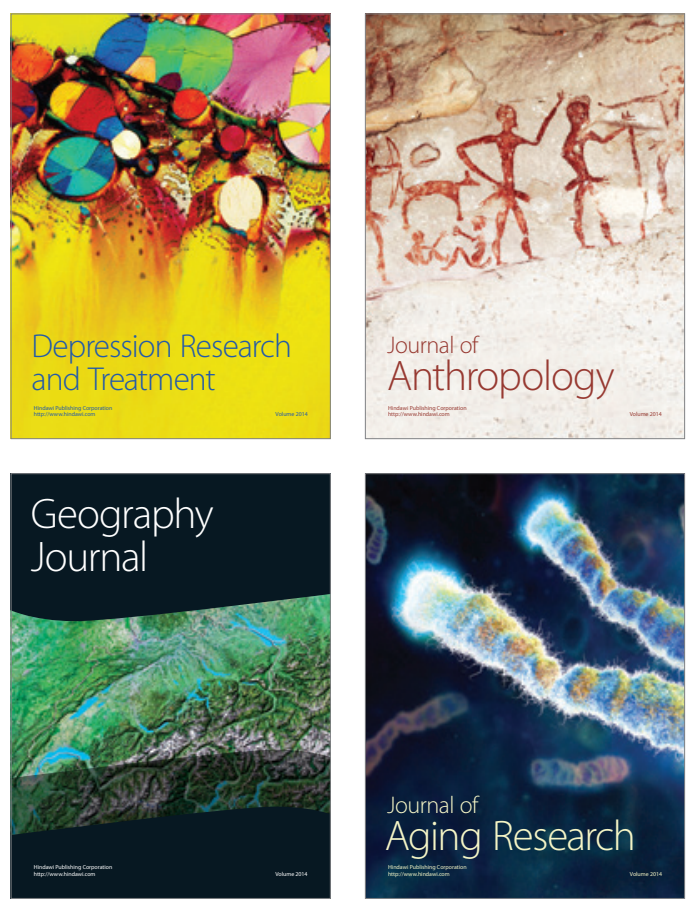
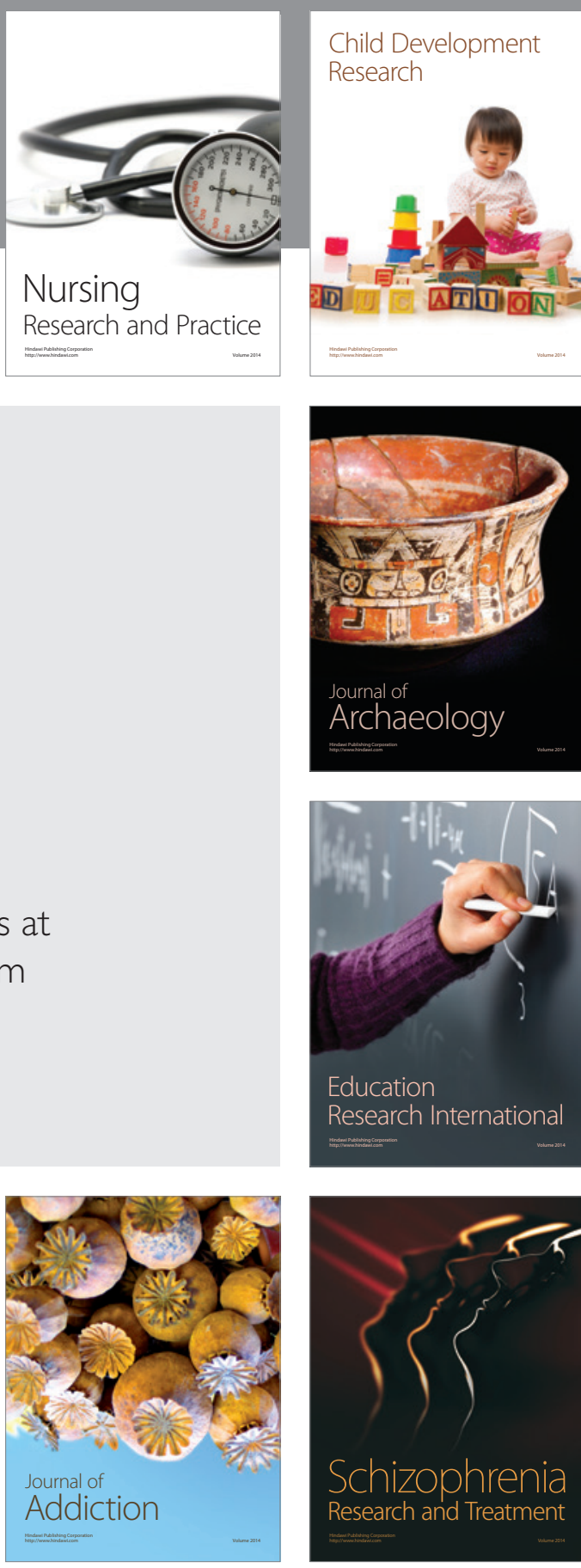

(D)
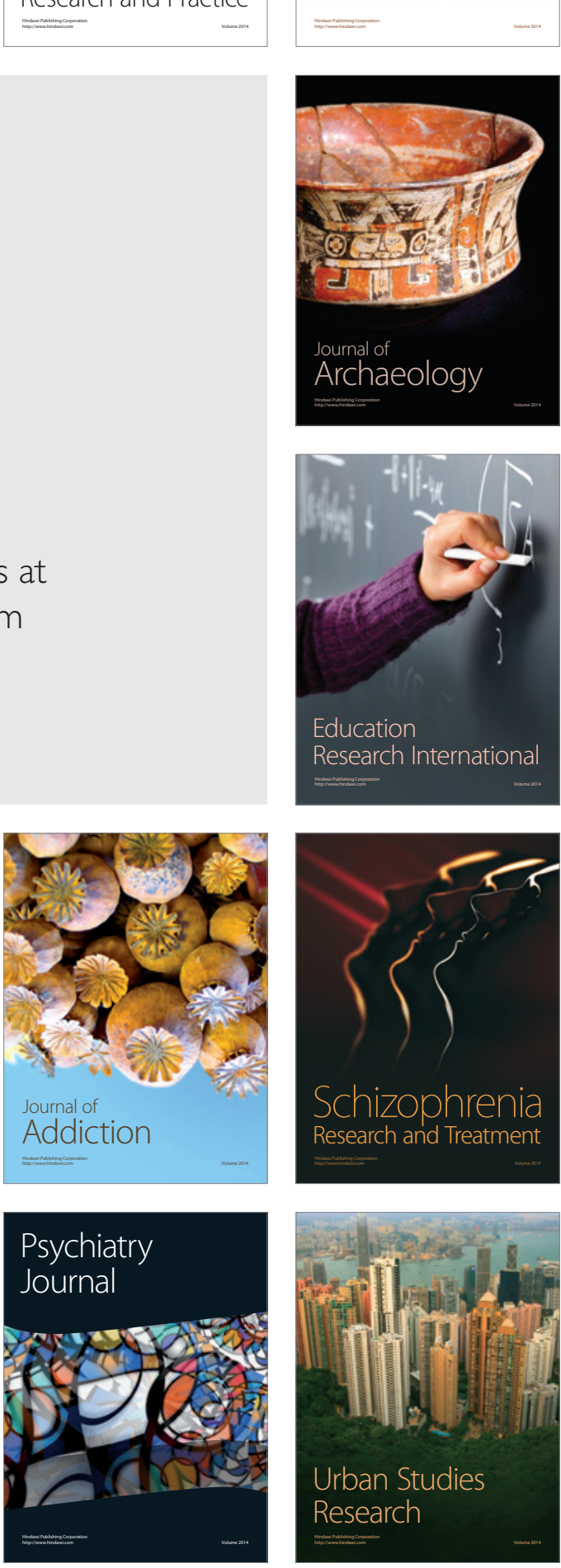